\title{
Assessment of Genetic Diversity Using Morpho-Agronomical Traits in Horse Gram
}

\author{
Sunil Shriram Gomashe ${ }^{1 *}$, Nilamani Dikshit ${ }^{1}$, Dinesh Chand ${ }^{1}$ and Smita N. Shingane ${ }^{2}$ \\ ${ }^{1}$ ICAR-National Bureau of Plant Genetic Resources, Regional Station, Dr. PDKV Campus, \\ Akola-444 104, Maharashtra, India \\ ${ }^{2}$ Post Graduate Institute, Mahatma Phule Krishi Vidyapeeth, Rahuri-413722, \\ Maharashtra, India \\ *Corresponding author
}

\begin{tabular}{|l|}
\hline Ke y w o r d s \\
GCV, PCV, Diversity \\
analysis, Shannon \\
$\begin{array}{l}\text { Diversity index, } \\
\text { Euclidean distances }\end{array}$ \\
\hline Article Info \\
\hline $\begin{array}{l}\text { Accepted: } \\
\text { 16 April } 2018 \\
\text { Available Online: } \\
\text { 10 May } 2018\end{array}$ \\
\hline
\end{tabular}

\section{A B S T R A C T}

Horse gram [Macrotyloma uniflorum (Lam.) Verd.] is one of the highly nutritious minor pulse crop and has number of desirable traits like drought tolerance, high protein content, antioxidant activity, antimicrobial and medicinal properties. A total of 66 genotypes of horse gram comprising 61 accessions of indigenous collections belonging to 15 states of India, one exotic collection from Australia and four checks were evaluated in Randomized Block Design (RBD) consecutively for three years [Kharif seasons of 2013 (E1), 2014 (E2) and 2015 (E3)] at ICAR-National Bureau of Plant Genetic Resources (ICARNBPGR), Regional Station, Akola, Maharashtra. Accessions were evaluated for seven each of qualitative and quantitative traits. Significant variability was observed among the accessions for most of the quantitative traits. Highest coefficient of variation was observed in seed yield per plant (g) followed by pods per plant and pod length. The phenotypic coefficients of variation (PCV) were higher than the genotypic coefficient of variation (GCV) for all the traits. The highest magnitude of PCV and GCV were recorded for seed yield per plant $(\mathrm{g})$ and pods per plant followed by plant height $(\mathrm{cm})$. Shannon diversity index (SDI) varied from 0.078 to 0.686 showed existence of sufficient variability among the accessions for qualitative traits. Accessions were classified on the basis of Euclidean distances using Ward's method. The dendrogram revealed two major clusters. Cluster I comprised of 17 accessions and cluster II comprised of 49 accessions. Cluster II was further classified in two sub-clusters (IIa and IIb). Most of the accessions from Tamil Nadu were confined to cluster I indicating their similarity for quantitative traits. Whereas accessions from rest of the states were well distributed in cluster II exhibiting high diversity for the traits studied. The accessions from cluster I could be used for hybridization program with the accessions from cluster II in order to develop superior horse gram genotypes/varieties for overall yield enhancement.

\section{Introduction}

Horse gram (Macrotyloma uniflorum (Lam.) Verdc.) is an important minor legume crop has its origin in India (Bogdan, 1977). It is mainly cultivated in the localities of underprivileged resource poor farmers in central and southern region (Chhatisgarh, Jharkhand, Maharashtra, 
Odisha, Telangana, Andra Pradesh, Karnataka and Tamilnadu) of the country. It has very high nutritional value which forms a very quality protein source to the farmers in tribal areas (Dikshit et al., 2014). Horse gram grains contains up to $27 \%$ protein (Kumar, 2007) high in lysine content compared to other pulses such as chick pea and red gram (Yadav, 2004). Taxonomically it belongs to the family Fabaceae with diploid chromosome number $(2 \mathrm{n}=20, \quad 22, \quad 24) . \quad$ It has self-fertile cleistogamous flowers producing pods with 46 grains (Cook et al., 2005). Clinical studies reported that horse gram has extraordinary therapeutic properties and in our traditional ayurvedic treatments it used to treat the ailments related to kidney stones, urinary diseases, piles, cardiac problems, asthma etc. It has high dietary fibre content which is very useful for good bowel movements and reduction of symptoms related to obesity (Kumar, 2007; Bhartiya et al., 2015). Horse gram is also a very good source of iron and molybdenum micronutrients and overall chemical composition of grains is as good as other cultivated legumes (Vishwanatha et al., 2016). This crop has also high fodder value due to its protein richness and it is majorly used to feed milch animals and horses (Cook et al., 2005; Bhadana et al., 2013). Agronomically, it is very important for sustainable farming with its atmospheric nitrogen fixation ability. It also helps in reduction of soil erosion with good canopy cover over the soil. Horse gram crop takes about $120-180$ days to mature. It is usually cultivated in resource poor soils as a rainfed crop. In paddy growing areas it is broadcasted immediately after the harvest of paddy so as to take maximum advantage of residual soil moisture. It is very hardy and drought tolerant crop. Very few efforts have been made for the improvement of this minor but very important pulse crop. High variability existed in horse gram germplasm for various traits. The utilization of this information for improvement is of high significance. The present investigation was targeted to study the genetic variability among the horse gram accessions collected from different states of India and to assess the correlation among the different traits of interest and drawing of inferences for utilization in the horse gram improvement.

\section{Materials and Methods}

A total of 66 horse gram genotypes comprising 61 accessions of indigenous collections from about 15 states of India, one exotic collection from Australia and four checks (Table 1) were evaluated at ICARNational Bureau of Plant Genetic Resources (ICAR-NBPGR), Regional Station, Akola, Maharashtra in Randomized Block Design (RBD) consecutively for three years [Kharif seasons of 2013 (E1), 2014 (E2) and 2015 (E3)]. Recommended package of practices were followed to raise a good crop. Sowing was done with $90 \times 30 \mathrm{~cm}$ crop geometry. Each genotype was sown in 3 rows of 3 meter length. The observations on seven quantitative traits viz., plant height $(\mathrm{cm})$, primary branches per plant, pods per plant, pod length $(\mathrm{cm})$, seeds per pod, 100 seed weight $(\mathrm{g})$ and seed yield per plant $(\mathrm{g})$ were recorded on five random plants. For qualitative traits visual scoring was done using standard descriptors for horse gram. Quantitative data was analysed using Windostat software to work out variability parameters. Diversity analysis using Euclidean distances (Wards`method) was carried out with Paleontological Statistics Software Package $f$ or Education and Data Analysis (PAST) (Hammer et al., 2001) software (Ver. 3.14) freely available at www.folk.uio.no/ohammer/past/.

\section{Results and Discussion}

Horse gram with its importance as functional food is gaining attention worldwide 
specifically in under developed and developing countries. In developed nations it is an important food for dietary fibres. There are various advantages of growing horse gram for its capabilities to fix atmospheric nitrogen, drought tolerance and cover crop to check soil erosion. It is mainly grown on resource poor soils with negligence of improved production technologies. Farmers use the remnant seeds to cultivate this crop which results in poor performance and reduction in overall productivity. Keeping this in view, a wellplanned strategy is required to assess the genetic variability in horse gram for various important agronomic attributes, identification of association between the traits and effect of numerous traits on yield levels. The present investigation was also carried out to address these issues. A total 66 horse gram accessions were evaluated for three years and mean performance and variability parameters were assessed for further utilization in crop improvement programme. Significant differences were observed among the accessions for most of the traits except pod length $(\mathrm{cm})$ and seeds per pod (Table 2). Highest coefficient of variation was observed in seed yield per plant followed by pods per plant and pod length (Parameshwarappa and Kumar 2002; Gupta et al., 2010; Kulkarni and Mogle, 2011). Among the 66 genotypes, IC 277625 (7.00), IC 277630 (6.70) and IC 145250 (6.63) recorded more primary branches per plant. For pods per plant, IC 53627 (49.60), IC 26132 (49.43) and IC 9623 were superior. Pod length $(\mathrm{cm})$ and seeds per pod were almost similar in all the accessions studied. Tall plant types was observed in the accessions IC $277630(90.65 \mathrm{~cm})$ followed by IC $277677(87.04 \mathrm{~cm})$ and IC 277625 (83.49 $\mathrm{cm})$. Superior genotypes for seed yield per plant (g) were IC 341308 (19.51 g), IC 145250 (13.66 g) and IC 22783 (13.29 g). Accessions, IC $139527(3.59 \mathrm{~g})$, IC $15735(3.57 \mathrm{~g})$ and IC277677 (3.36g) recorded highest 100 seed weight (g) (Table 2). Similar observations on agro-morphological traits were reported by Prakash and Khanure (2000); Prakash et al., 2008; Prakash et al., 2010; Sunil et al., 2014 and Vishwanatha et al., 2016 in horse gram.

\section{Genetic variability estimates}

The phenotypic coefficients of variation (PCV) were higher than the genotypic coefficient of variation (GCV) for all the traits. The characters seed yield per plant, pods per plant, plant height and 100 seed weight exhibited high estimates of GCV, PCV and ECV (Sunil et al., 2008; Gupta et al., 2010). The heritability estimates were also high for seed yield per plant, 100 seed weight and plant height. Genetic advance $\%$ of mean at 5\% was high for seed yield per plant and pods per plant (Table 3 ).

\section{Diversity for qualitative traits}

Shannon diversity index varied from 0.078 to 0.686 showed existence of sufficient variability among the accessions (Table 4). High values for Shannon Diversity Index (SDI) were reported for the traits growth pattern, leaf surface, stem colour and pod surface. This indicated richness of this population for diverse phenotypes available for these traits. Sankar et al., 2015 reported high SDI for Per cent disease Index (PDI) trait in horse gram.

\section{Diversity in quantitative traits}

Accessions were classified in to different clusters on the basis of Euclidean distances using Ward's method. The dendrogram revealed two major clusters. Cluster I comprised of 17 accessions and cluster II comprised of 49 accessions. Cluster II was further classified in two sub-clusters (IIa and IIb). Most of the accessions from Tamil Nadu were confined to cluster I indicating their similarity for quantitative traits. 
Table.1 List of horse gram accessions with passport information

\begin{tabular}{|c|c|c|c|c|c|c|c|}
\hline SN & IC/EC No. & State & Country & SN & IC/EC No. & State & Country \\
\hline 1 & IC 9623 & Sikkim & India & 34 & IC 89010 & Tamil Nadu & India \\
\hline 2 & IC 9624 & Sikkim & India & 35 & IC 105588 & Jharkhand & India \\
\hline 3 & IC 9626 & Sikkim & India & 36 & IC 121640 & Himachal Pradesh & India \\
\hline 4 & IC 10938 & Karnataka & India & 37 & IC 123030 & Tamil Nadu & India \\
\hline 5 & IC 15735 & Jharkhand & India & 38 & IC 123033 & Bihar & India \\
\hline 6 & IC 15775 & Manipur & India & 39 & IC 139331 & Maharashtra & India \\
\hline 7 & IC 19552 & Odisha & India & 40 & IC 139527 & Andhra Pradesh & India \\
\hline 8 & IC 22750 & Madhya Pradesh & India & 41 & IC 139544 & Uttarakhand & India \\
\hline 9 & IC 22783 & Chhattisgarh & India & 42 & IC 145245 & Himachal Pradesh & India \\
\hline 10 & IC 23444 & Chhattisgarh & India & 43 & IC 145250 & Himachal Pradesh & India \\
\hline 11 & IC 23510 & Chhattisgarh & India & 44 & IC 145268 & Gujarat & India \\
\hline 12 & IC 26132 & Karnataka & India & 45 & IC 264704 & Kerala & India \\
\hline 13 & IC 26135 & Manipur & India & 46 & IC 277625 & Karnataka & India \\
\hline 14 & EC 28842 & - & Australia & 47 & IC 277630 & Karnataka & India \\
\hline 15 & IC 45748 & Tamil Nadu & India & 48 & IC 277659 & Karnataka & India \\
\hline 16 & IC 47119 & Andhra Pradesh & India & 49 & IC 277670 & Karnataka & India \\
\hline 17 & IC 47129 & Andhra Pradesh & India & 50 & IC 277671 & Karnataka & India \\
\hline 18 & IC 49290 & Maharashtra & India & 51 & IC 277677 & Karnataka & India \\
\hline 19 & IC 49555 & Maharashtra & India & 52 & IC 277687 & Karnataka & India \\
\hline 20 & IC 53627 & Chhattisgarh & India & 53 & IC 282589 & Jharkhand & India \\
\hline 21 & IC 55065 & Manipur & India & 54 & IC 321300 & Tamil Nadu & India \\
\hline 22 & IC 68593 & Kerala & India & 55 & IC 336492 & Bihar & India \\
\hline 23 & IC 71725 & Tamil Nadu & India & 56 & IC 341308 & Maharashtra & India \\
\hline 24 & IC 71730 & Tamil Nadu & India & 57 & IC 343105 & Jharkhand & India \\
\hline 25 & IC 71743 & Tamil Nadu & India & 58 & IC 343190 & Chhattisgarh & India \\
\hline 26 & IC 71763 & Tamil Nadu & India & 59 & IC 347181 & Jharkhand & India \\
\hline 27 & IC 71766 & Tamil Nadu & India & 60 & IC 385839 & Jharkhand & India \\
\hline 28 & IC 71770 & Tamil Nadu & India & 61 & IC 561038 & Jharkhand & India \\
\hline 29 & IC 71781 & Tamil Nadu & India & 62 & IC 561040 & Jharkhand & India \\
\hline 30 & IC 71783 & Tamil Nadu & India & 63 & $\mathrm{AK}-21$ & Maharashtra & India \\
\hline 31 & IC 71803 & Tamil Nadu & India & 64 & AK-26 & Maharashtra & India \\
\hline 32 & IC 71817 & Tamil Nadu & India & 65 & HGGP & - & India \\
\hline 33 & IC 71825 & Tamil Nadu & India & 66 & PHG-9 & - & India \\
\hline
\end{tabular}


Int.J.Curr.Microbiol.App.Sci (2018) 7(5): 2095-2103

Table.2 Mean performance of horse gram genotypes for various traits

\begin{tabular}{|c|c|c|c|c|c|c|c|c|}
\hline SN & IC/EC No. & PRI_BRN & POD_PLT & POD_LT & PLT_HGT & SED_POD & YLD_PLT & SED_WGT \\
\hline 1 & IC 9623 & 5.90 & 48.47 & 4.34 & 57.13 & 4.43 & 7.65 & 3.03 \\
\hline 2 & IC 9624 & 5.03 & 28.20 & 3.98 & 47.74 & 3.90 & 4.26 & 2.53 \\
\hline 3 & IC 9626 & 5.53 & 19.67 & 4.08 & 65.39 & 4.03 & 3.76 & 2.83 \\
\hline 4 & IC 10938 & 5.97 & 24.27 & 4.21 & 58.56 & 4.00 & 8.52 & 2.64 \\
\hline 5 & IC 15735 & 5.90 & 31.97 & 4.34 & 64.93 & 4.33 & 3.66 & 3.57 \\
\hline 6 & IC 15775 & 5.63 & 30.97 & 4.05 & 62.86 & 3.90 & 3.16 & 3.29 \\
\hline 7 & IC 19552 & 5.03 & 26.10 & 4.35 & 61.22 & 4.13 & 7.93 & 2.96 \\
\hline 8 & IC 22750 & 5.97 & 26.23 & 4.09 & 57.30 & 4.20 & 5.44 & 2.64 \\
\hline 9 & IC 22783 & 6.23 & 46.43 & 4.10 & 61.25 & 4.35 & 13.29 & 3.07 \\
\hline 10 & IC 23444 & 5.27 & 29.90 & 4.24 & 66.69 & 4.27 & 5.93 & 3.26 \\
\hline 11 & IC 23510 & 6.22 & 35.23 & 4.27 & 70.15 & 4.63 & 9.71 & 3.14 \\
\hline 12 & IC 26132 & 5.83 & 49.23 & 4.40 & 66.36 & 4.23 & 9.93 & 2.89 \\
\hline 13 & IC 26135 & 6.03 & 29.50 & 4.16 & 63.80 & 4.33 & 6.21 & 3.11 \\
\hline 14 & EC 28842 & 5.57 & 23.60 & 4.65 & 74.33 & 4.53 & 4.95 & 3.08 \\
\hline 15 & IC 45748 & 5.13 & 28.33 & 4.32 & 60.32 & 4.50 & 5.23 & 2.89 \\
\hline 16 & IC 47119 & 5.80 & 39.40 & 4.25 & 60.59 & 4.37 & 5.96 & 3.28 \\
\hline 17 & IC 47129 & 5.50 & 29.57 & 5.66 & 65.33 & 4.33 & 6.04 & 3.15 \\
\hline 18 & IC 49290 & 5.57 & 43.03 & 4.05 & 63.90 & 3.97 & 3.92 & 2.30 \\
\hline 19 & IC 49555 & 5.37 & 31.60 & 4.20 & 53.16 & 4.30 & 5.11 & 2.39 \\
\hline 20 & IC 53627 & 5.47 & 49.60 & 4.27 & 57.45 & 3.97 & 6.50 & 3.04 \\
\hline 21 & IC 55065 & 5.60 & 46.07 & 4.24 & 64.23 & 4.83 & 6.38 & 2.88 \\
\hline 22 & IC 68593 & 5.93 & 36.97 & 4.46 & 68.03 & 4.47 & 7.03 & 3.01 \\
\hline 23 & IC 71725 & 6.47 & 41.70 & 3.80 & 68.32 & 3.93 & 3.26 & 3.18 \\
\hline 24 & IC 71730 & 5.87 & 24.93 & 4.52 & 60.90 & 4.50 & 3.67 & 2.60 \\
\hline 25 & IC 71743 & 6.13 & 33.43 & 4.17 & 54.63 & 4.07 & 8.75 & 2.97 \\
\hline 26 & IC 71763 & 5.93 & 39.63 & 4.35 & 55.30 & 4.57 & 5.88 & 3.12 \\
\hline 27 & IC 71766 & 5.47 & 32.27 & 4.59 & 55.68 & 4.53 & 3.24 & 2.52 \\
\hline 28 & IC 71770 & 5.17 & 31.60 & 4.53 & 59.97 & 4.50 & 3.68 & 2.44 \\
\hline 29 & IC 71781 & 5.13 & 31.60 & 4.22 & 50.47 & 4.37 & 3.40 & 2.59 \\
\hline 30 & IC 71783 & 5.13 & 26.43 & 4.55 & 55.15 & 4.13 & 3.35 & 2.50 \\
\hline 31 & IC 71803 & 5.63 & 22.27 & 4.43 & 52.75 & 4.20 & 3.73 & 2.96 \\
\hline 32 & IC 71817 & 6.00 & 27.23 & 4.76 & 51.27 & 4.63 & 3.48 & 2.52 \\
\hline 33 & IC 71825 & 5.10 & 35.53 & 4.64 & 51.98 & 4.27 & 4.46 & 2.68 \\
\hline 34 & IC 89010 & 5.63 & 22.50 & 4.56 & 71.60 & 4.30 & 3.29 & 3.05 \\
\hline 35 & IC 105588 & 5.60 & 24.53 & 4.41 & 59.79 & 3.80 & 6.06 & 2.61 \\
\hline 36 & IC 121640 & 5.67 & 24.43 & 4.02 & 46.39 & 3.90 & 3.45 & 2.82 \\
\hline 37 & IC 123030 & 5.43 & 23.57 & 3.96 & 52.96 & 3.87 & 3.75 & 3.01 \\
\hline 38 & IC 123033 & 5.92 & 34.03 & 4.40 & 57.71 & 4.20 & 7.81 & 2.85 \\
\hline 39 & IC 139331 & 6.43 & 32.33 & 4.23 & 61.17 & 4.50 & 8.09 & 3.34 \\
\hline 40 & IC 139527 & 6.50 & 44.60 & 4.24 & 74.37 & 4.77 & 11.71 & 3.59 \\
\hline
\end{tabular}




\begin{tabular}{|c|c|c|c|c|c|c|c|c|}
\hline 41 & IC 139544 & 5.57 & 26.00 & 4.45 & 61.55 & 4.10 & 7.69 & 2.86 \\
\hline 42 & IC 145245 & 6.03 & 17.73 & 4.05 & 50.65 & 3.72 & 6.39 & 2.85 \\
\hline 43 & IC 145250 & 6.63 & 41.47 & 4.50 & 60.66 & 3.77 & 13.66 & 2.99 \\
\hline 44 & IC 145268 & 5.77 & 28.60 & 4.36 & 58.80 & 4.33 & 10.44 & 3.11 \\
\hline 45 & IC 264704 & 6.70 & 32.73 & 4.41 & 63.80 & 3.80 & 9.13 & 2.88 \\
\hline 46 & IC 277625 & 7.00 & 27.83 & 4.33 & 83.49 & 4.33 & 4.13 & 3.26 \\
\hline 47 & IC 277630 & 6.97 & 38.53 & 4.32 & 90.65 & 4.57 & 6.53 & 3.10 \\
\hline 48 & IC 277659 & 6.57 & 19.53 & 4.26 & 81.32 & 4.13 & 3.75 & 2.98 \\
\hline 49 & IC 277670 & 5.87 & 16.13 & 4.20 & 73.26 & 3.57 & 2.96 & 2.81 \\
\hline 50 & IC 277671 & 6.67 & 19.40 & 4.24 & 77.29 & 4.33 & 5.52 & 3.18 \\
\hline 51 & IC 277677 & 6.13 & 22.17 & 4.12 & 87.04 & 4.18 & 2.97 & 3.36 \\
\hline 52 & IC 277687 & 5.93 & 25.00 & 4.11 & 76.27 & 4.30 & 4.01 & 3.12 \\
\hline 53 & IC 282589 & 5.87 & 31.73 & 4.35 & 61.60 & 4.43 & 5.85 & 2.76 \\
\hline 54 & IC 321300 & 6.00 & 24.83 & 4.27 & 66.20 & 4.50 & 4.57 & 3.13 \\
\hline 55 & IC 336492 & 5.90 & 21.73 & 4.16 & 75.21 & 4.13 & 4.16 & 3.20 \\
\hline 56 & IC 341308 & 5.83 & 44.67 & 4.42 & 73.11 & 4.37 & 19.51 & 3.40 \\
\hline 57 & IC 343105 & 5.50 & 22.30 & 4.47 & 66.91 & 4.17 & 4.37 & 3.04 \\
\hline 58 & IC 343190 & 5.73 & 26.77 & 4.28 & 56.54 & 3.93 & 6.40 & 3.01 \\
\hline 59 & IC 347181 & 5.73 & 30.17 & 4.02 & 63.07 & 4.00 & 5.00 & 2.69 \\
\hline 60 & IC 385839 & 5.97 & 25.90 & 4.20 & 72.96 & 4.00 & 5.65 & 3.06 \\
\hline 61 & IC 561038 & 5.93 & 15.47 & 4.28 & 65.94 & 3.97 & 3.22 & 2.93 \\
\hline 62 & IC 561040 & 6.07 & 27.67 & 4.06 & 72.81 & 4.17 & 4.69 & 2.76 \\
\hline 63 & AK-21 & 6.37 & 33.70 & 4.36 & 71.33 & 4.23 & 3.79 & 3.08 \\
\hline 64 & AK-26 & 5.27 & 26.07 & 4.43 & 56.64 & 4.47 & 11.45 & 3.17 \\
\hline 65 & HGGP & 5.90 & 34.73 & 4.15 & 63.28 & 4.37 & 3.70 & 2.90 \\
\hline 66 & PHG-9 & 5.57 & 15.30 & 4.27 & 74.32 & 4.37 & 6.23 & 3.14 \\
\hline & Mean & 5.84 & 30.35 & 4.31 & 63.88 & 4.23 & 6.02 & 2.96 \\
\hline & Minimum & 5.03 & 15.30 & 3.80 & 46.39 & 3.57 & 2.96 & 2.30 \\
\hline & Maximum & 7.00 & 49.60 & 5.66 & 90.65 & 4.83 & 19.51 & 3.59 \\
\hline & C.D. $5 \%$ & 0.90 & 15.11 & NS & 14.81 & NS & 3.57 & 0.40 \\
\hline & C.D. $1 \%$ & 1.19 & 19.90 & NS & 19.51 & NS & 4.71 & 0.53 \\
\hline
\end{tabular}

PRI_BRN $=$ Primary branches per plant; POD_PLT $=$ Pods per plant; POD_LT $=$ pod length $(\mathrm{cm})$; PLT_HGT $=$ Plant height $(\mathrm{cm})$; SED_POD= Seeds per pod; YLD_PLT = Seed yield per plant $(\mathrm{g})$; SED_WGT $=100$ seed weight $(\mathrm{g})$

Table.3 Variability estimates for different traits in horse gram

\begin{tabular}{|l|r|r|r|r|r|r|r|}
\hline Parameters & PRI_BRN & POD_PLT & POD_LT & PLT_HGT & SED_POD & YLD_PLT & SED_WGT \\
\hline ECV & 13.63 & 43.83 & 13.65 & 20.41 & 16.31 & 52.25 & 11.88 \\
\hline GCV & 5.45 & 21.65 & 1.64 & 11.92 & 2.28 & 46.18 & 7.97 \\
\hline PCV & 14.68 & 48.89 & 13.75 & 23.64 & 16.15 & 69.73 & 14.30 \\
\hline h' (B S.) & 0.13 & 0.19 & 0.01 & 0.25 & -0.02 & 0.43 & 0.31 \\
\hline $\begin{array}{l}\text { GA as \% of } \\
\text { Mean 5\% }\end{array}$ & 4.17 & 19.75 & 0.40 & 12.39 & -0.66 & 63.00 & 9.15 \\
\hline
\end{tabular}

PRI_BRN = Primary branches per plant; POD_PLT = Pods per plant; POD_LT = pod length $(\mathrm{cm})$; PLT_HGT $=$ Plant height $(\mathrm{cm})$; SED_POD= Seeds per pod; YLD_PLT $=$ Seed yield per plant $(\mathrm{g})$; SED_WGT $=100$ seed weight $(\mathrm{g})$ 
Table.4 Variability in qualitative traits among 66 horsegram accessions

\begin{tabular}{|c|c|c|c|c|}
\hline Descriptors & Descriptor states & Frequency & Percent & SDI \\
\hline \multirow[t]{3}{*}{ Plant vigour } & Poor & 2 & 3.03 & \multirow[t]{3}{*}{0.501} \\
\hline & Good & 8 & 12.12 & \\
\hline & Very good & 56 & 84.85 & \\
\hline \multirow[t]{2}{*}{ Growth habit } & Bushy & 1 & 1.52 & \multirow[t]{2}{*}{0.078} \\
\hline & Semi spreading & 65 & 98.48 & \\
\hline \multirow{2}{*}{$\begin{array}{l}\text { Growth } \\
\text { Pattern } \\
\end{array}$} & Determinate & 26 & 39.39 & \multirow[t]{2}{*}{0.670} \\
\hline & Indeterminate & 40 & 60.61 & \\
\hline \multirow[t]{2}{*}{ Leaf surface } & Glabrous & 45 & 68.18 & \multirow[t]{2}{*}{0.625} \\
\hline & Pubescent & 21 & 31.82 & \\
\hline \multirow[t]{2}{*}{ Stem colour } & Green & 50 & 75.76 & \multirow[t]{2}{*}{0.554} \\
\hline & Purple & 16 & 24.24 & \\
\hline \multirow[t]{2}{*}{ Pod surface } & Glabrous & 16 & 24.24 & \multirow[t]{2}{*}{0.554} \\
\hline & Pubescent & 50 & 75.76 & \\
\hline
\end{tabular}

Table.5 Promising accessions identified for different traits in horse gram

\begin{tabular}{|r|l|l|}
\hline SN & \multicolumn{1}{|c|}{ Trait } & \multicolumn{1}{|c|}{ Promising accessions } \\
\hline $\mathbf{1}$ & Primary branches per plant & IC 277625, IC 277630, IC 145250 \\
\hline $\mathbf{2}$ & Pods per plant & IC 53627, IC 26132, IC 9623 \\
\hline $\mathbf{3}$ & Plant height (cm) & IC 277630, IC 277677, IC 277625 \\
\hline $\mathbf{4}$ & Seed yield per plant (g) & IC 341308, IC 145250, IC 22783 \\
\hline $\mathbf{5}$ & 100 seed weight (g) & IC 139527, IC 15735, IC 277677
\end{tabular}

Fig.1 Grouping of 66 horse gram accession on the basis of Euclidean distances

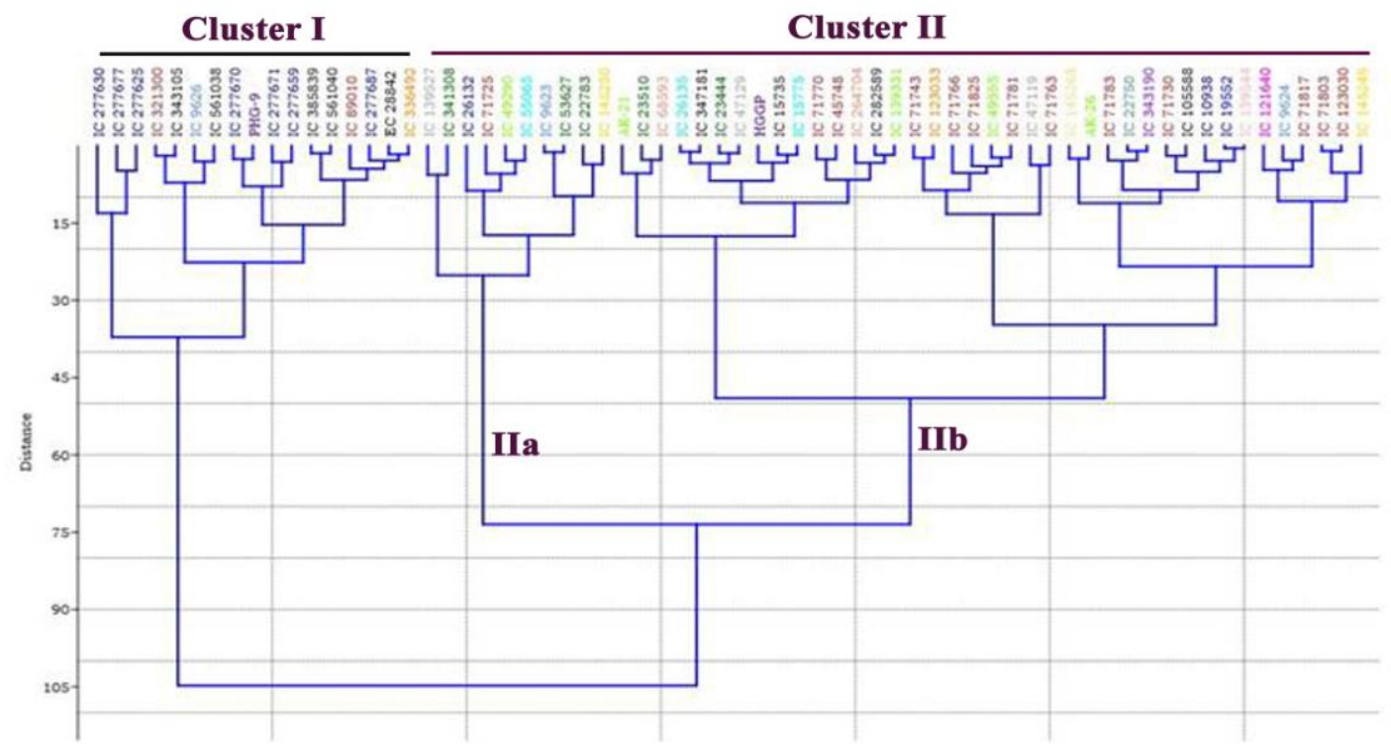


Whereas accessions from rest of the states were well distributed in cluster II exhibiting high diversity for the traits studied. The accessions from cluster I could be used for hybridization program with the accessions from cluster II in order to develop high yielding genotypes/varieties for overall yield enhancement (Fig. 1). Sunil et al., 2008 and Vishwanatha et al., 2016 also reported clustering of horse genotypes into different clusters and also reported important and distant accessions for utilization in horse gram crop improvement (Gupta et al., 2010). Promising accessions for different traits are reported (Table 5) and these can be used for improvement of the specific traits in horse gram.

\section{References}

Bhadana, V.P., P.K. Sharma, M.A. Ansari, L.K. Baishya, P. Punetha, S. Datt, N. Prakash, and Rana, K.S. 2013. Food legumes for livelihood and nutritional security in North Eastern Himalayan region: Prospects and constraints. Indian Journal of Agricultural Science. 83(9):899-906.

Bhartiya, J. P. Aditya and Kant L. 2015. Nutritional and remedial potential of an underutilized food legume horse gram (Macrotyloma uniflorum): A review. The Journal of Animal \& Plant Sciences. 25(4): 908-920.

Bogdan, A.V. 1977. Tropical pastures and fodder plants. pp. 475. Longman, London

Cook, B.G., B.C. Pengelly, S.D. Brown, J.L. Donnelly, D.A. Eagles, M.A. Franco, J. Hanson, B.F. Mullen, I.J. Partridge, M. Peters, and Schultze-Kraft, R.2005. Tropical Forages: an interactive selection tool. [CD-ROM], CSIRO, DPI\&F (Qld), CIAT and ILRI, Brisbane, Australia.
Dikshit, N., G. Katna, C. S. Mohanty, A. B. Das and Sivaraj, N. 2014. Horse gram. In M. Singh et al., (eds.), Broadening the Genetic Base of Grain Legumes. Springer India. pp. 209-215

Gupta, A., Bhartiya, A., Singh, G., Mahajan, V. and Bhatt, J.C. 2010. Altitudinal diversity in horse gram (Macrotyloma uniflorum (Lam.) Verdc.) landraces collected from Himalayan hill region. Plant Genetic Resources: characterization and utilization. 8: 214216.

Hammer, O. D., A. Harper and P. D. Ryan. 2001. Paleontological Statistics Software Package for Education and Data Analysis. www.paleeoelectronica. org/2001-1/past/issue1_01.htm.

Kulkarni GB. 2010. Evaluation of genetic diversity of horse gram (Macrotyloma uniflorum) germplasm through phenotypic trait analysis. Green Faarming 1(6):563-565.

Kulkarni, G.B. and Mogle U. P.2011. Characterization and Evaluation of Horse Gram [Macrotyloma uniflorum (Lam)] Verdcourt Genotypes. Science Research Reporter. 1(3):122-125.

Kumar, D. 2007. Production technology for horse gram in India. ICAR-Central Arid Zone Research Institute, Jodhpur, Rajasthan. Pp.1-20.

Parameshwarappa, S.G, and Kumar, D.L. 2002. Performance of horsegram cultivars on medium black soils of Northern transitional zone of Karnataka. Karnataka Journal of Agriculture Sciences. 15:627-629.

Prakash, B. G., C. P. Hiremath., S.B. Devarnavdgi and Salimath, P. M. 2010. Assessment of genetic diversity among germplasm lines of horsegram (Macrotyloma uniflorum L.) at Bijapur. Electronic Journal of Plant Breeding. 1:414-419. 
Prakash, B.G. and Khanure, S. 2000. Genetic parameters, correlation and path coefficient analysis in horsegram. Karnataka Journal of Agriculture Sciences. 13:312-314.

Sankar A. U., K. Anitha, N. Sivaraj, K.V.S. Meena Kumari., N. Sunil and Chakrabarty, S.K.2015. Screening of horse gram germplasm collected from Andhra Pradesh against anthracnose. Legume Research. 38 (6): 753-757.

Sunil, N., N. Sivaraj, S.R. Pandravada, V. Kamala, P. R. Reddy and Varaprasad, K.S. 2008. Genetic and geographical divergence in horsegram germplasm from Andhra Pradesh, India. Plant Genetic Resources Characterization and Utilization. 7:84-87.
Sunil, N., Vinod Kumar, S. Natarajan, V. Kamala and Pandravada S. R. 2014. Evaluation and diversity observed in Horsegram (Macrotyloma uniflorum (Lam) Verdc.) germplasm from Andhra Pradesh. India International Journal of Plant Research 2014. 4(1): 17-22.

Viswanatha, K.P., L.N. Yogeesh and Amitha, K. 2016. Morphological diversity study in horsegram (Macrotyloma uniflorum (Lam.) Verdc) based on Principal Component Analysis (PCA) Electronic Journal of Plant Breeding. 7(3): 767770.

Yadav, S. 2004. Protein and oil rich wild horsegram. Genetic Resources and Crop Evolution. 51:629-633.

\section{How to cite this article:}

Sunil Shriram Gomashe, Nilamani Dikshit, Dinesh Chand and Smita N. Shingane. 2018. Assessment of Genetic Diversity Using Morpho-Agronomical Traits in Horse Gram. Int.J.Curr.Microbiol.App.Sci. 7(05): 2095-2103. doi: https://doi.org/10.20546/ijcmas.2018.705.244 